\title{
Arbitrage forcé et justice d'État pendant la Révolution française d'après l'exemple de Montpellier
}

"Arbitrage forcé" and State Justice during the French Revolution: the Example of Montpellier

Carine Jallamion

\section{OpenEdition Journals}

\section{Édition électronique}

URL : https://journals.openedition.org/ahrf/11248

DOI : 10.4000/ahrf.11248

ISSN : 1952-403X

\section{Éditeur :}

Armand Colin, Société des études robespierristes

\section{Édition imprimée}

Date de publication : 1 décembre 2007

Pagination : 69-85

ISSN : 0003-4436

Référence électronique

Carine Jallamion, « Arbitrage forcé et justice d'État pendant la Révolution française d'après l'exemple de Montpellier », Annales historiques de la Révolution française [En ligne], 350 | octobre-décembre 2007 mis en ligne le 01 janvier 2011, consulté le 01 juillet 2021. URL : http://journals.openedition.org/ahrf/ 11248 ; DOI : https://doi.org/10.4000/ahrf.11248 


\title{
ARBITRAGE FORCÉ ET JUSTICE D'ÉTAT PENDANT LA RÉVOLUTION FRANÇAISE D'APRÈS L'EXEMPLE DE MONTPELLIER
}

\author{
Carine JALLAMION
}

\begin{abstract}
Par méfiance envers la justice étatique mais aussi par foi en ce mode alternatif de règlement des litiges, les révolutionnaires expérimentent dès 1790 l'arbitrage forcé, rendu obligatoire essentiellement en matière familiale. La loi vient ainsi réglementer l'institution de l'arbitrage par nature contractuelle et extrajudiciaire, d'abord par touches légères, puis au fur et à mesure que la Révolution se radicalise, l'on voit les domaines où il est obligatoire d'avoir recours à des arbitres se multiplier, et la nature de l'institution profondément évoluer, d'extrajudiciaire à judiciarisée, à tel point que le législateur semblant vouloir la substituer à la justice traditionnelle de la Nation, lui impose des contraintes telles qu'elle finit par lui ressembler en tout point et se trouver ainsi complètement dénaturée.
\end{abstract}

Mots-clés : arbitrage, tribunaux de famille, justice, procédure, équité, successions.

Larbitrage est par nature un mode extrajudiciaire de règlement des litiges. Il représente une alternative à la justice étatique, dans la mesure où les parties sont libres de choisir cette voie et de l'organiser comme elles l'entendent par le biais du contrat. Elles peuvent ainsi désigner leurs arbitres, prévoir la mission qu'elles confient à ces derniers, qui ne sont pas en principe tenus de suivre les règles de procédure ni les règles de fond qui s'imposent aux juges. Les parties peuvent également décider que la sentence sera rendue en dernier ressort, sans aucun recours possible à la justice

(1) Cet article constitue la reprise d'un des aspects de notre travail de thèse, L'arbitrage en matière civile du XVII" au XIX' siècle. L'exemple de Montpellier, Thèse de droit, Montpellier, 2004, 535 p. 
étatique, auquel cas l'arbitrage est alors pleinement extrajudiciaire. L'arbitrage n'est toutefois pas complètement distinct de la justice traditionnelle car l'arbitre exerce aussi une mission juridictionnelle. Larbitre tient en effet sa légitimité et son pouvoir de la convention des parties, mais une fois investi et dans les limites de l'affaire qui lui est soumise, il exerce une véritable jurisdictio, au sens où il dit le droit et tranche le litige comme le ferait le juge ${ }^{2}$.

Cette double nature, extrajudiciaire et juridictionnelle de l'arbitrage, constitue l'un de ses avantages, car elle autorise plus de souplesse que dans le procès ordinaire. Elle fait donc bien de l'arbitrage une alternative à la justice de la Nation, et non une autre justice qui lui ressemblerait en tout point. Cette affirmation, que révèlent à la fois la nature de l'arbitrage et son histoire, se trouve pourtant contredite lors des premières années de la Révolution française, précisément de la loi des 16-24 août 1790 jusqu'à la loi du 9 ventôse an IV, quand est tentée l'expérience de l'arbitrage forcé, celui auquel les parties doivent obligatoirement se soumettre quand leur litige touche à tel domaine du droit déterminé par le législateur. Larbitrage forcé n'est plus alors une alternative à la justice car il n'y a plus de choix entre l'une ou l'autre voie. Mais l'examen de la pratique montpelliéraine révèle que dans un premier temps, malgré le cadre législatif imposé qui sur quelques points vient se substituer à la volonté des parties, les arbitres persistent à demeurer, en accord avec les litigants, dans le domaine de l'extrajudiciaire. Tel est l'arbitrage rendu par les tribunaux de famille institués par la loi des 16-24 août 1790. Puis, quelques mois après l'installation de la Convention et par défiance envers les juges, le pouvoir entend faire de l'arbitrage un substitut à la justice étatique ainsi régénérée. Pour y parvenir, le législateur décide de multiplier les arbitrages forcés et de ne plus laisser désormais aux parties la possibilité d'organiser l'arbitrage ni de prévoir ses effets. Trop réglementé, l'arbitrage finit alors par ressembler à ce de quoi il devait à tout prix se distinguer et éviter de répéter les travers : la justice étatique elle-même, dont il n'est plus qu'une modalité.

\section{L'arbitrage des tribunaux de famille : une réalité extrajudiciaire}

La faveur des révolutionnaires pour l'arbitrage se révèle rapidement, au cours de la réforme de la justice dont le chantier commence dès le mois d'août 1789 , et elle est corrélative de la méfiance des révolutionnaires envers la justice traditionnelle. La réforme entreprise par les Constituants aboutit ainsi à la loi des 16-24 août 1790 qui contient deux séries de dispositions sur l'arbitrage, lesquelles reposent sur la foi quasi dogmatique du

(2) Cf. Dominique Bureau et Charles Jarrosson, "Arbitrage ", dans Denis Alland et Stéphane Rials (dir.), Dictionnaire de la culture juridique, Paris, PUF, 2003, p. 76. 
pouvoir politique dans la résolution extrajudiciaire des conflits. Le bon sens de l'arbitre devra ainsi permettre un meilleur règlement du conflit que ne l'aurait fait le juge et plus généralement le juriste, car l'arbitre n'a pas à justifier de connaissances juridiques pour exercer ses fonctions et doit plutôt juger en équité ${ }^{3}$. La réforme s'inscrit donc dans un projet plus vaste de régénération d'une justice devant fonctionner avec le moins possible de juristes et de règles de droit ${ }^{4}$.

Le titre $I^{\text {er }}$ de la loi des 16-24 août présente d'abord l'arbitrage volontaire comme « le moyen le plus raisonnable de terminer les contestations entre les citoyens $»^{5}$. À ceux qui s'étonnent de voir ainsi figurer un mécanisme extrajudiciaire en tête d'une loi consacrée à la réforme des institutions judiciaires, l'avocat et député Thouret, rapporteur du texte, répond que « la justice des tribunaux n'est instituée que comme un remède extrême pour ceux qui n'ont pas l'esprit de s'en passer $"$ ". Il est donc logique que l'arbitrage précède la justice, et dans le même esprit un préliminaire de conciliation devant le juge de paix est aussi imposé?. Ce dernier figure au titre $\mathrm{X}$ de la loi, titre dans lequel l'on trouve aussi l'institution des tribunaux de famille, auxquels époux et parents doivent obligatoirement avoir recours pour régler leurs différends.

Cette idée d'un arbitrage obligatoire à l'intérieur du cercle de la famille n'est pas nouvelle et a notamment déjà été formulée par l'un des deux édits de Fontainebleau pris par François II sur les conseils de son chancelier Michel de L'Hospital, en août $1560^{\circ}$. Ce texte instituait en effet un arbitrage obligatoire entre parents, pour les partages de successions, les comptes de tutelle et les restitutions de dot et de douaire" car, disait le texte, il s'agit de choses « plus de fait que de droict ", et qu'il est nécessaire d'« entretenir paix et amitié entre proches parents». Dans ces sortes de litiges, les parents en conflit devaient choisir des " arbitres " qui soient aussi « de bons et notables personnages jusqu'à trois parens, amis ou voisins ». Lédit précisait enfin que la sentence avait « force de chose jugée »,

(3) Aucune exigence en ce sens n'est en effet posée dans le décret des 16-24 août 1790. Cf. JeánLouis Halpérin, " La composition des tribunaux de famille sous la Révolution ou "Ies juristes, comment s'en débarrasser?" ", La famille, la loi, l'État de la Révolution au Code civil, Paris, éd. Centrc Gcorges Pompidou, 1989, p. 293.

(4) Voir sur ce point, Jean-Louis HaLPÉRIN, L'impossible Code civil, Paris, PUF, 1992, Coll. Histoires, p. 89.

(5) Décret des 16-24 août 1790, Titre I", art. 1 ${ }^{\circ t}$, Duvergiek, Collection complète des lois, décrets, ordonnances, règlements et avis du conseil d'État, t. 1, p. 310.

(6) Thouret, 16 août 1790 , dans J. MAVIDAL et L. LAURENT (dir.), Archives parlementaires (AP), $1^{\text {tore }}$ série, XVIII, p. 89.

(7) Décret des 16-24 août 1790, Titre X, art. 2, Duvergier, op. cit., t. I, p. 326. t. XIV,p. 51

(8) Édit de Fontaincbleau, août 1560, ISAMBERT, Recueil général des anciennes lois françaises,

(9) Pour les « divisions et partages de successions et biens communs de père ou mère, ayeulx, ayeulles, et enfans des enfans, frères, socurs, oncles, et d'enfans de frères et sceurs, et comptes de tutèles, et autres administrations, restitutions de dot et douaire entre lesdites personnes ", ISAMBERT, op. cit., I. XIV, p. 52. 
bénéficiait de l'exécution provisoire et pouvait faire l'objet d'un appel au parlement ${ }^{11}$.

Lédit de Fontainebleau n'a pas en son temps manqué de susciter l'étonnement et la critique des auteurs d'Ancien Régime qui affirment la nature nécessairement volontaire de l'arbitrage, par exemple par la voix de l'avocat parisien Pigeau, fin connaisseur de la procédure, qui rappelle que " quoique les loix aient fait très sagement d'établir l'arbitrage dans ces cas [partage, compte de tutelle, restitution de dot et douaire], on ne peut disconvenir que si les parties n'y ont recours que malgré elles, cette voie peut bien leur être avantageuse, mais elle ne le sera jamais autant que si elles la prennent de leur gré " ". Les parlements n'ont d'ailleurs pas enregistré le texte ${ }^{12}$ et les parties elles-mêmes ne s'y sont pas soumises, préférant l'arbitrage volontaire qui leur permettait tous les aménagements possibles $^{13}$.

Pourtant, malgré cet échec, le législateur révolutionnaire reprend l'idée à son compte, par la voix de Thouret pour qui les tribunaux de famille permettront d'« étouffer sans éclat les contestations de deux époux ou de proches parents qui, sans cela, après avoir scandalisé la société, finissent quelquefois par opérer la ruine d'une famille entière $"^{14}$. Le projet de Thouret est voté dans l'enthousiasme, malgré les réticences de Robespierre qui dénonce la partialité de cette justice familiale ${ }^{15}$. Il aboutit ainsi à l'article 12 du titre $X$ de la loi des 16-24 août 1790, qui veut que «s'il s'élève quelque contestation entre mari et femme, père et fils, grand-père et petitfils, frères et sœurs, neveux et oncles, ou entre alliés aux degrés ci-dessus, comme aussi entre pupilles et leurs tuteurs pour choses relatives à la tutelle, les parties seront tenues de nommer des parens, amis ou voisins pour arbitres, devant lesquels ils éclairciront leur différend, et qui, après les avoir entendues et avoir pris les connaissances nécessaires, rendront une décision motivée ». Il est ensuite précisé que chaque partie nommera deux arbitres, lesquels pourront être désignés d'office par le juge du tribunal de district en cas de refus. Également, « la partie qui se croira lésée par la

(10) Ibid.

(11) Pigenu, La procédure civile du Châtelet, Paris, 1779, t. I, p. 17.

(12) Nous renvoyons sur ce point à notre thèse, op. cit., Deuxième partie, «Larbitrage volontaire en concurrence ", Titre I, "La pratique volontaire de l'arbitrage forcé ", Chapitre I, "Lidée de l'arbitrage obligatoire entre parents ". Section I, "Une idćc ancienne : l'édit de 1560 ", p. 210 et sq.

(13) Nous renvoyons là encore à notre thèse, op. cit., Premièrc partic, "Larbitrage volontaire en héritage ", p. 17 et sq.

(14) Thouret, 5 août 1790, $A P, 1^{\text {tre }}$ série, t. XVII, p. 616.

(15) ROHE.spttekR: fait en effet valoir que l'institution est « contraire à tous les principes " ainsi qu' impraticable " : "vous voulez que le frère, plaidant contre son frère, soit jugé comme les autres citoyens; vous voulez que le jugement soit également impartial. L'institution qu'on vous propose renferme tous les germes de la partialité : les jugements ne seraient plus rendus suivant la justice, mais suivant l'affection des juges pour les parties; $2^{\circ}$ Comment espérez-vous que toutes les familles seront assez nombreuses pour fournir des juges?" ", 5 août $1790, A P, 1$ "série, t. XVII, p. 621. 
décision arbitrale, pourra se pourvoir par appel devant le tribunal de district, qui prononcera en dernier ressort $»^{16}$, l'arbitrage se trouvant ainsi soumis au contrôle de la justice étatique.

Par la suite, la compétence de ces tribunaux est encore étendue signe de la confiance que le législateur place toujours dans cette institution - lorsque, par le décret des 20-25 septembre 1792, qui vient pour la première fois autoriser le divorce en droit français, les " arbitres de famille ${ }^{17}$ se voient confier la dernière des trois procédures mises en place par la loi, celle du divorce pour cause déterminé $e^{i k}$. Les arbitres devront simplement ici constater l'existence d'un des sept motifs de séparation prévus par le législateur, soit «1. La démence, la folie ou la fureur de l'un des époux ; 2. La condamnation de l'un d'eux à des peines afflictives ou infamantes ; 3. Les crimes, sévices ou injures graves de l'un envers l'autre ; 4. Le dérèglement des mours notoire ; 5 . Labandon de la femme par le mari ou du mari par la femme, pendant deux ans au moins ; 6. L'absence de l'un d'eux, sans nouvelles, pendant au moins cinq ans ; 7. Lémigration dans les cas prévus par la loi ${ }^{19}$; et une fois «ces faits vérifiés », les époux seront renvoyés devant l'officier d'état civil « pour faire prononcer le divorce ${ }^{21}$.

Les tribunaux de famille se trouvent ainsi soumis à des règles impératives, prévues par le législateur et non par les parties, qui tiennent à la fois aux conditions, aux modalités et aux effets de l'arbitrage. Mais la contrainte se veut encore assez légère et, en pratique, tant les arbitres que les litigants vont, sous couvert d'arbitrage forcé, procéder d'une manière qui ressemble à s'y méprendre à l'arbitrage volontaire tel que pratiqué traditionnellement depuis l'Ancien Régime pour régler les différends familiaux. Sur ce point, la législation révolutionnaire ne marque donc pas encore une rupture et, malgré sa soumission au législateur et au contrôle de la justice, l'arbitrage conserve dans la pratique son caractère extrajudiciaire.

L'on remarque d'abord qu'à Montpellier, les parties se soumettent volontiers à l'arbitrage des tribunaux de famille, car rares sont les cas où l'une des parties doit demander l'assistance du juge de district afin d'obtenir

(16) Décret des 16-24 août 1790, Tître X, art. 12, 13 ct 14, DuVErgier, op. cit.. t. I, p. 326.

(17) Décret des 20-25 septembre 1792, art. 18, DUVERGIER, op. cit., 1. IV, p. 480.

(18) Deux autres procédures sont en effet également proposées aux époux : le divorce par consentement mutuel et le divorce pour incompatibilité d'humeur, dans lesquels interviennent des assemblées de parents et non des arbitres.

(19) Décret des 20-25 septembre 1792, art. 4, DUVERGILR, op. cit., t. IV, p. 477.

(20) Ibid., art. 19, DuVERGIER, op. cit., t. IV, p. 480. Voir également le décret des 8 et 14 nivôse an II, préambule, art. 2 et art. 9, DUVeroieR, op. cit., t. VI. p. 359-340 ; et le décret des 4 et 9 floréal an II, DUVERGIER, op. cil., t. VII, p. 152. 
désignation d'office des arbitres pour la partie défaillante ${ }^{21}$, et rares sont également les procédures menées par défaut ${ }^{22}$.

Les parties doivent d'abord choisir leurs arbitres. Sur ce point, la loi est claire, exigeant que chaque partie en désigne deux, ce qui correspond aussi à la tradition d'Ancien Régime. Les parties s'y conforment donc sans peine. Les arbitres doivent également avoir la qualité de parents, amis ou voisins, et ici, les litigants ne se plient que rarement aux prescriptions législatives $^{23}$, préférant désigner des hommes de loi. À Montpellier, sont ainsi choisis des « gens de loi $»^{24}$, des $«$ hommes de loi $»^{25}$, des avoués auprès du tribunal local ${ }^{26}$, des greffiers ${ }^{27}$. Comme sous l'Ancien Régime, les mêmes individus sont souvent choisis, révélant une certaine professionnalisation de l'arbitrage : à Montpellier Jean-Edmond Serres ${ }^{28}$ et Caizergues, tous deux juristes, sont par exemple en 1792 systématiquement désignés dans une affaire sur trois. La présence de juristes au sein des tribunaux de famille est en effet rendue nécessaire par le fait que la qualité des parties n'emporte pas la simplicité du litige, et qu'au contraire les différends qui naissent en matière familiale, particulièrement en matière successorale, sont par nature fort complexes.

Les parties s'octroient aussi une certaine liberté en ce qui concerne le déroulement de l'instance arbitrale. La loi des 16-24 août 1790 prévoit que les parties, devant le tribunal de famille, "éclairciront leur différend ", et que les arbitres, « après les avoir entendues et avoir pris les connaissances nécessaires, rendront une décision motivée ${ }^{29}$. Le législateur indique également qu'en cas de désaccord entre les arbitres, eux-mêmes désigneront un tiers afin de les départager ${ }^{30}$. Ce cadre contraignant n'empêche pas les parties d'aménager la mission qu'elles confient aux arbitres, par exemple en prévoyant, comme c'était souvent le cas aux siècles précédents, l'intervention de tiers en tant que médiateurs, qui deviendront éventuelle-

(21) Cf. par exemple AD Hérault, L 6332, 2 décembre 1790, Requête de Jean Savajol et jugement pour Jean Savajol père contre son fils.

(22) Cf. par exemple AD Hérault, L 6333, 17 février 1891, Sentence arbitrale rendue entre Pierre Roudier et Pierre Treilles. Une procédure par défaut est légèrement plus fréquente dans les affaires de divorce : cf. par cxemple AD Hérault, L. 6334, 22 décembre 1792, Décision motivéc pour Marie Desfours contre Laurent Hérail.

(23) Cf. par exemple AD Hérault, L 6333, 17 février 1791, Sentence arbitrale entre Pierre Roudier et Pierre Treilles.

(24) Cf. AD Hérault, L 6333, 7 avril 1791 et 6 juin 1792, Décision motivée pour Jean-Baptiste contre Louis Espinas.

(25) Cf. AD Hérault, L 6333, 9 octobre 1791, Décision motivée pour les sieurs Arnaud père et fils.

(26) Cf. AD Hérault, L 6335, 31 mai 1793, Décision motivée entre Elisabeth Durand contre Mathieu Oustet.

(27) Cf. AD Hérault, L 6333, 9 octobre 1791, Décision motivée pour les sieurs Arnaud père et fils.

(28) Jean-Edmond Serres est très certainement avocat mais aussi professeur à la faculté de droit de Montpellier.

(29) Décret des 16-24 aoút 1790, Titre X, art. 12, Duvergier, op. cit., t. I. p. 326-327.

(30) Ibid., art. 13. 
ment arbitres en cas d'échec de la conciliation. Ainsi dans une sentence rendue le 2 mai 1792 entre un frère et sa sœur, les arbitres inscrivent en tête de leur sentence qu'ils composent « le tribunal de famille afin de concilier s'il se peut le sieur Gros et sa sœur au sujet de leur différend, sinon rendre leur décision motivée conformément au titre 10 de la loi du 24 aoust 1790 ». Ils constatent ensuite qu'après « avoir employé sans succès la voye de la conciliation, nous avons été dans la nécessité de rendre notre décision motivée comme il suit " ${ }^{31}$. Un véritable arbitrage est ainsi finalement rendu. Plus souvent encore, les arbitres procèdent de même en matière de divorce, alors que la loi des 20-25 septembre 1792 les cantonne presque à un simple rôle d'expertise. Ainsi dans la procédure menée les 24 et 25 décembre 1792 entre les époux Galibert, les arbitres tentent en vain une conciliation, demandée par le mari, mais l'épouse demanderesse s'y opposant, ils convoquent les époux au lendemain pour constater le bien-fondé du motif de divorce $^{32}$.

Une fois les parties entendues et les pièces examinées, les arbitres délibèrent. La loi ne précisant rien sur ce point, les arbitres vont perpétuer des pratiques existant déjà sous l'Ancien Régime et rendre soit un arbitrage au sens strict, en délibérant seuls et en imposant leur solution aux parties, soit au contraire chercher à obtenir leur accord ou un arrangement. Lon voit ainsi des arbitres rendre un véritable arbitrage dans une sentence prononcée à Montpellier le 26 janvier 1791 entre Roussel et Doux, l'acte constatant que les arbitres ont «fait retiré lesdittes parties afin de [se] concilier librement sur toutes les questions des demandes et deffenses réciproques qui sont d'une assez grande importance dans leur décision ${ }^{33}$. Il arrive aussi que les arbitres procèdent de même mais prennent encore le soin d'obtenir l'acquiescement de la partie condamnée à la décision qu'ils viennent de rendre, ce que l'arbitrage ne requiert pas par principe puisque les litigants s'engagent par avance à exécuter la sentence arbitrale, mais c'est une précaution utile fréquemment rencontrée aux siècles précédents. Ainsi dans l'affaire jugée entre Antoine et Louise Gros le 2 mai 1792, les arbitres délibèrent seuls, mais « avant la dresse de la présente [la partie condamnée] en a eu connaissance, [et] a déclaré aux arbitres qu'elle entendait y acquiescer $[\ldots] \aleph^{34}$.

D'autres fois, l'on voit les arbitres délibérer à l'inverse en présence des parties et discuter avec elles la solution du litige, faisant alors primer

(31) AD Hérault, L. 6334, 2 mai 1792, Décision motivée entre le sieur Gros et la citoyenne Gros sa sueur.

(32) AD Hérault, L 6334, 24 et 25 décembre 1792, Procès verbal tenu par le tribunal de famille formé par la citoyenne Galihert et son époux.

(33) AD Hérault, L 6333, Sentence arbitrale du 26 janvier 1791 entre Roussel et Doux.

(34) AD Hérault, L 6334, 2 mai 1792, Décision motivée entre le sieur Gros et la citoyenne Gros sa sour. 
l'efficacité du règlement sur le strict respect des catégories juridiques. C'est par exemple le cas dans l'affaire survenue en 1791 entre un père et son fils, au sujet d'une donation de terre agricole faite par le premier au second, sous réserve que chaque année, une part de la récolte revienne au donateur. Parce que le père soupçonne son fils de sous-estimer la récolte, il forme un tribunal de famille devant lequel son fils demande un arrangement, afin que la part à donner à son père soit fixée en nature ou en argent. Devant les arbitres et d'après leur «avis », les parties parviennent à se faire " des propositions réciproques ", si bien qu'elles transigent finalement devant le tribunal de famille, dans un acte pourtant qualifié de « décision motivée ", c'est-à-dire de sentence du tribunal arbitral ${ }^{35}$.

Les délibérations du tribunal de famille permettent aussi, dans la mesure où la loi impose que les sentences soient motivées, de connaitre les règles d'après lesquelles les arbitres se déterminent. Comme auparavant, ils s'accordent le droit de statuer suivant les règles de droit ou en équité, profitant de ce que le législateur n'ait rien précisé sur ce point. Ainsi dans un arbitrage rendu entre deux frères en 1791, Louis Espinas demande à Jean-Baptiste, héritier universel de leur mère, l'exécution d'un legs et le remboursement d'une somme d'argent. Le défendeur reconnaît les faits et les arbitres le condamnent au paiement, mais parce qu'il ne peut pas acquitter sa dette immédiatement, il demande devant les arbitres si son frère accepterait de recevoir en paiement un immeuble de la succession, et à défaut que les arbitres lui accordent un délai. Les arbitres relèvent alors qu'en droit, le légataire « ne peut être contraint de recevoir en payement des immeubles de l'hérédité maternelle, ni pour le montant de l'obligation [...] parce qu'elle dérive d'un prêt fait en argent, ni pour le montant de ses droits légitimaires, parce que la testatrice lui a légué pour cet objet une somme fixe $[\ldots]$ qui doit être par conséquent payée en argent non en immeubles ». Mais notent-ils, suivant l'équité, parce qu'« il est juste d'avoir égard à la situation [de] Jean Baptistes Espinas qui est dans l'impossibilité de payer actuellement lesdites deux sommes, et qu'il convient de lui accorder un délai suffisant pour pouvoir vendre les immeubles avec les prix desquels il entend se libérer envers son frère », les arbitres accordent au défendeur un délai de six mois ${ }^{36}$.

Lon constate ainsi que l'intervention du législateur et la mise en place d'un arbitrage forcé ne modifient pas des pratiques multiséculaires et propres à la famille, des pratiques marquées par une certaine confusion juridique entre l'arbitrage et la conciliation mais qui correspondent au besoin des parties et au travail des arbitres eux-mêmes. Ainsi l'arbitrage

(35) AD Hérault, L 6333, 9 octobre 1791, Décision motivéc pour Arnaud père et fils.

(36) AD Hérault, L 6333, 7 avril 1791 et 6 juin 1792, Décision motivéc pour Jean-Baptiste Louis Espinas. 
forcé des lois des 16-24 août 1790 et des 20-25 septembre 1792 garde sa nature d'arbitrage, malgré le fait qu'il soit imposé. Rendant obligatoire l'arbitrage dans un domaine où il était pratiqué de tradition, la loi n'est pas venue modifier la pratique, et cet arbitrage demeure, malgré la possibilité de l'appel, extrajudiciaire. Il est bien et encore une alternative à la justice de la Nation, et voulu comme tel. Mais ce mouvement va quelque peu s'emballer avec la Convention qui va généraliser l'arbitrage forcé à partir de 1793. Un vrai désir va naître au sein du pouvoir de substituer l'arbitrage à la justice de l'État, mais faisant cela, les révolutionnaires vont imposer les mêmes règles contraignantes à l'arbitrage que celles qui existent pour la justice, et vont ainsi faire de l'arbitrage une autre justice d'État, en le dénaturant.

\section{La radicalisation de l'arbitrage forcé à partir de 1793 : l'arbitrage substitué à la justice de l'État}

La Convention nationale devient la nouvelle assemblée de la France le 21 septembre 1792, dans un contexte politique de plus en plus défavorable à la justice étatique, accusée de trahir la Révolution, particulièrement depuis le 10 août $1792^{37}$. La radicalisation du pouvoir politique et sa défiance des juges entraînent ainsi le vote d'une série de décrets, comme celui du 3 brumaire an II, qui vient réduire au strict minimum les formes à suivrc pour l'instruction des affaires et qui supprime les fonctions d'avoué ${ }^{38}$. En réaction à un pouvoir judiciaire qu'ils croient dévoyé, les Conventionnels choisissent de développer encore davantage l'arbitrage forcé. Signe de la volonté politique de le substituer à la justice étatique, la Constitution du 24 juin 1793 va jusqu'à qualifier les juges d' ' arbitres publics $»^{34}$.

La loi du 10 juin 1793 incarne d'abord cette tendance. Quand la Convention décide d'autoriser par ce texte le partage des biens communaux selon des modalités nouvelles, elle prévoit aussi que tous les litiges qui naîtront de l'application de la réforme seront obligatoirement réglés, non plus par la justice étatique, voie pourtant choisie par la précédente assemblée $^{410}$, mais par l'arbitrage. Le revirement du législateur s'explique en

(37) Cf. Jean-Jacques CLÈRE, « Larbitrage révolutionnaire : apogée et déclin d'une institution (1790)-1806) ", Revue de l'arhitrage, 1981, p. 16.

(38) Décret du 3 brumaire an Il (24 octobre 1793), DuvERGIER, op. cit., t. VI, p. 250-252. Voir sur cette question Claudine Bloch et Jcan HILAIRE, "Nouveauté et modernité du droit révolutionnaire : la procédure civilc ", La Révolution et l'ordre juridique privé, rationalité ou scandale? Actes du colloque d'Orléans, 11-13 septembre 1986, Paris, PUF, 1988, t. II, p. 468.

(39) Acte constitutionnel et déclaration des droits de l'homme du 24 juin 1793, De la justice civile, art. 91 à 95, DUVERGIFr, op. cit., t. V, p. 356-357.

(40) Le décret des 28 auút-4 septembrc 1792 portait en effet en son art. 2 al. 1 que " pour rentrer en possession des portions de leurs biens communaux dont elles ont été privées par l'effet de ladite ordonnance et desdits édits et déclarations, arrêts, lettres parentes, jugemens et actes, les communautés seront tenues de se pourvoir, dans l'espace de cinq ans, par devant les tribunaux [...] ", Duverciler, op. cit., t. IV, p. 376 . 
grande partie par l'inertie des juges, âprement dénoncée par Fabre de l'Hérault lors de la discussion du projet, qui relève que "les communes ne devraient jamais plaider ; elles sont toujours mal défendues ; personne n'apportant, dans la poursuite de leurs affaires, la chalcur de l'intérêt particulier, elles sont suivies avec lenteur, et souvent jugées avec inattention : les frais en sont toujours énormes. Les procès entre communautés sont à présent l'unique ressource de l'avide praticien : mémoires volumineux, consultations multipliées, requêtes inutiles, rien n'est épargné pour prolonger des procédures qui forment un revenu annuel à d'industrieux avoués [...] Obliger les communes à plaider, c'est décréter leur ruine. Leur permettra-t-on de vendre pour cet objet leurs biens patrimoniaux ? Bientôt ils seront consumés en procédures, et il ne restera plus rien aux habitants qu'un procès qu'on aura l'art de prolonger [...] Car, il ne faut pas se le dissimuler, la justice, quoique gratuite, est encore bien chère et la chicane n'a rien perdu de son activité. Si donc les procès que font naître de toutes parts les biens communaux se poursuivent devant les tribunaux ordinaires, le partage n'est plus qu'un mot dont on cherchera vainement la réalité. Déjà, de toutes parts, il nous arrive des pétitions, pour demander la permission d'emprunter ou de vendre pour fournir aux frais de procédures ${ }^{41}$.

Un tel constat, qui est aussi un aveu d'échec, amène logiquement selon Fabre de l'Hérault à l'arbitrage, " le moyen le plus simple et le plus utile de déterminer ces procès ", rappelant que " cette vérité sert de base à la loi sur l'organisation judiciaire ${ }^{42}$. Son projet aboutit ainsi à la loi du 10 juin 1793, par laquelle le législateur décide que chaque fois que le litige portera sur autre chose que le mode de partage des biens communaux ${ }^{43}$, la voie de l'arbitrage devra être obligatoirement choisie pour parvenir à son règlement, à la fois entre communes et propriétaires ou usurpateurs, ainsi qu'entre communes ${ }^{44}$.

À propos de la procédure arbitrale, Fabre de l'Hérault avait indiqué lors de la présentation du texte: « Nous ne nous sommes point égarés dans les formes : quelques articles simples et clairs ont suffi $n^{45}$. Le législateur prévoit ainsi que les parties en litige doivent se rendre " devant le juge de paix du canton », lequel recevra la désignation d'« un ou plusieurs arbitres " par les parties, mais toujours "à nombre égal " ${ }^{4 h}$. La parité exigée pour la

(41) Fabre de l'Hérault, séance du 8 avril 1793, $A P$, 1're série, t. LXI, p. 435-436.

(42) Ibid.

(43) Décret des 10-11 juin 1793, section V, art. 1" et 2, DUVERGIER, op. cit., t. V. p. 331.

(44) Ihid., art. 3, 4 et 5 . Voir les articles 3 à 5 de la Section VI du projet présenté par Fabre de l'Hérault, séance du 8 avril 1793, $A P, 1$ "re série, t. LXI, p. 441. Voir également la séance du 10 juin 1793, $A P$, $1^{\text {ter }}$ série, t. LXVI, p. 229-230.

(45) Fabre de l'Hérault, séance du 8 avril $1793, A P, 1^{\text {tre }}$ série, t. LXI, p. 436.

(46) Décret des 10-11 juin 1793, section V, art. 6, Duvergiter, op. cit., t. V, p. 332. Le décret du 2 octobre 1793 vient ensuite réduirc le nombre des arbitres qui peuvent être désignés à trois par intérêt en présence, Décret du 2 octobre 1793, art. 2 et 3, DUVERGIER, op. cit., t. VI, p. 204. 
composition des tribunaux de famille n'est ainsi plus obligatoire, et, plutôt que le juge de district, le juge de paix est choisi pour assister et contrôler le bon déroulement de l'arbitrage. Ce dernier doit ensuite dresser procèsverbal de la désignation ${ }^{47}$, et éventuellement nommer d'office les arbitres pour la partie qui s'y refuserait ${ }^{48}$. Il est également prévu que les parties disposent d'un mois pour remettre " leurs titres et mémoires entre les mains des arbitres », lesquels devront rendre leur sentence « deux mois après cette remise $~_{49}{ }^{49}$. Le législateur réglemente aussi l'éventuel recours par les arbitres à des experts, qui en pratique sera souvent nécessaire, experts nommés par les arbitres ${ }^{51}$ et devant procéder en présence des parties ${ }^{5 !}$. Une fois les preuves accumulées et les vérifications effectuées, la loi précise que les arbitres devront déterminer les « droits, usages, prétentions, demandes en rétablissement dans les propriétés dont [les communes] ont été dépouillées par l'effet de la puissance féodale ». Or il s'agit d'autant de points sur lesquels il a été légiféré, ce qui signifie, et c'est une rupture majeure dans l'histoire de l'arbitrage, que les arbitres ont désormais obligation de suivre la loi et ne peuvent plus juger en équités2. Enfin, si les arbitres ne parviennent pas à se mettre d'accord, ils s'adresseront au bureau de paix du canton qui désignera un tiers-arbitre ${ }^{53}$. Quant aux effets de la sentence, il est prévu qu'elle sera " exécutée sans appel, et rendue exécutoire par une simple ordonnance du président du tribunal de district ${ }^{54}$. Un système de récusation des arbitres sera tout de même instauré trois mois plus tard ${ }^{55}$. Pour l'instant, Fabre de l'Hérault se satisfait de telles dispositions et prévoit que « dans trois mois, tous les procès pour les communaux seront terminés $"^{5 / 2}$.

Cet arbitrage est ainsi plus encore qu'auparavant presque complètement soustrait à la volonté des parties pour être étroitement contrôlé par le législateur, soit directement, soit par l'intermédiaire du juge de paix. Lévolution du législateur témoigne ici d'une grande méfiance, voire d'une défiance, à l'égard à la fois des parties, des arbitres et des juges. La défiance à l'égard des parties empêche de les laisser libres d'aménager l'instance arbitrale à leur convenance. La défiance à l'égard des arbitres

(47) Décret des 10-11 juin 1793, section V, art. 7 et 8, DUVERGIER, op. cit., t. V, p. 332.

(48) lbid., art. 9 et 10.

(49) Ibid, art. 12.

(50) lbid., art. 13.

(51) lbid., art. 14.

(52) Ce point fondamental est encore rappeié par le décret du 2 octobre 1793 qui précise que tous les procès qui pourraient s'élever à raison des biens communaux " seront vidés par la voie de l'arbitrage, et jugés d'après les principes établis par les décrets des 28 août 1792 et 10 juin dernier ", Décret du 2 octobre 1793, art. $1^{\text {"r}}$, DUVERGIER, op. cit., t. VI, p. 204.

(53) Décret des 10-11 juin 1793, section V, art. 15 à 18, Duvergier, op. cit., t. V, p. 333.

(54) lbid, art. 21.

(55) Décret du 2 octobre 1793, art. 4, 5 et 6, Duvergier, op. cit., t. VI, p. 204.

(56) Fabre de l'Hérault, séance du 8 avril 1793, $A P, 1^{\text {trt }}$ série, t. LXI, p. 436 et 441. 
interdit de les laisser maîtres du choix du tiers-arbitre, comme c'était le cas devant les tribunaux de famille, et ne permet plus de les laisser procéder cux-mêmes aux vérifications requises ou de régler le litige en équité. La défiance à l'égard des juges doit être certes relativisée, car seul le juge de district pâtit de l'attitude du législateur, tandis que le juge de paix paraît avoir au contraire toute la confiance des Conventionnels, les arbitres apparaissant d'ailleurs presque comme ses auxiliaires. Le dogme de la résolution extrajudiciaire des conflits a ainsi abouti à cette anomalie juridique : aussi réglementé que l'est la justice étatique, l'arbitrage ne laisse plus rien paraître d'extrajudiciaire ni de contractuel. Il devient ainsi une autre expression de la justice de l'État.

Les arbitrages rendus en vertu de la loi du 10 juin 1793 confirment d'ailleurs cette analyse, les arbitres s'y bornant le plus souvent à vérifier, de manière quasi administrative et avec l'aide d'experts, les limites des terres en fonction des plans fournis par les parties ${ }^{57}$. La loi a de ce point de vue produit une véritable rupture dans la pratique, car l'arbitrage a désormais une vocation nouvelle, selon le législateur : tout entier entre ses mains, il doit aboutir, comme la justice étatique, à une application mécanique de la loi.

Ce phénomène s'accentue encore avec le vote de la loi du 12 brumaire an II (2 novembre 1793) sur les droits des enfants « nés hors du mariage » et non adultérins ${ }^{5 x}$, puis de la loi du 17 nivôse an Il (6 janvier 1794) sur les partages de succession ${ }^{54}$. Ces lois « de combat " ${ }^{6(1)}$ présentent la particularité d'établir au profit des enfants naturels puis de tout héritier des droits égalitaires de succession, dont le règlement doit remonter de manière rétroactive au 14 juillet $1789^{51}$, disposition qui ne manquera pas de susciter un contentieux fort important que le législateur choisit une nouvelle fois de confier à des arbitres forcés.

S'agissant de la première de ces lois, alors que l'on aurait pu penser que le législateur ferait encore confiance aux tribunaux de famille, tel n'est pas l'esprit des Conventionnels, comme Cambacérès par exemple, pour qui " le danger des préventions [l]'empêche de proposer des arbitres de famille " Cette attitude témoigne d'une volonté de durcir l'arbitrage forcé, car il n'est plus question de l'abandonner à des arbitres qui en avaient permis la souplesse, dans un cadre législatif qui apparaît finalement assez peu contraignant au regard de ce qui allait suivre. Comme dans la loi du 10 juin

(57) Voir pour un exemple AD Hérault, L 6337. 19 nivôse an II, Procès verbal fait par les citoyens Bastide et Trouche.

(58) Décret du 12 brumaire an II, Duvergier, op. cit., t. VI, p. 269.

(59) Décret des 17-21 nivôse an II, DuverGier, op. cit., t. VI, p. 373.

(60) Cf. Jean-Louis HALPÉkIN, L'impossible Code civil..., op. cit., p. 143-167.

(61) Décret du 12 brumaire an II, art. 1 et 15, DUVERGIER, op. cit., t. VI, p. 269 et 271. Décret du 17 nivôse an II, art. 1`, DUVERGIER, op. cit., t. VI, p. 373. Voir sur cette question Jean-Louis HaLPERIN, L'impossible Code civil..., op. cil., p. 201-230, et Histoire des droits en Europe de 1750 à nos jours, Paris, Flammarion, 2004, p. 47.

(62) Rapport du 4 juin 1793, $A P$, $1^{\text {cer }}$ série, t. LIX, p. 35. 
1793 , le recours à des arbitres est rendu obligatoire ${ }^{13}$, le droit qu'ils devront appliquer - les règles de partage établies par le législateur au profit des enfants naturels ${ }^{64}$, et non l'équité - est également imposé ; enfin les effets de l'arbitrage sont par avance décidés, l'appel étant toujours impossible ${ }^{6.5}$.

Deux mois plus tard, la loi du 17 nivôse an II (6 janvier 1794) est aussi votée afin de poser de nouvelles règles de partage égalitaire des successions, cette fois à l'égard de tous les héritiers. La voie arbitrale est ici encore forcée, le texte précisant bien qu'il « est défendu aux tribunaux ordinaires d'en connaitre, et de donner suite [aux contestations] qui seraient

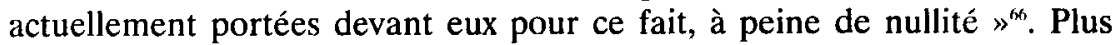
encore que dans les lois précédentes, le législateur impose ici de lourdes contraintes aux arbitres et aux parties.

Les parties sont d'abord obligées de choisir deux arbitres ${ }^{67}$, le juge de paix devant éventuellement procéder d'office ${ }^{\text {his }}$, et devant aussi désigner un tiers-arbitre en cas de partage ${ }^{(19}$. Le contentieux n'étant plus confié aux tribunaux de famille, il est entendu que les arbitres ne doivent pas être parents avec les parties, qualité qui constitue désormais une cause de récusation $^{70}$. Lc contrôle de la procédure repose encore davantage sur le juge de paix, justement compétent en matière de récusation des arbitres comme pour les "plaintes portées à raison de la négligence " de ces derniers". À Montpellier, les parties se soumettent sans difficulté à ces dispositions" ${ }^{2}$.

Les arbitres doivent également être munis, comme les professions judiciaires, d'un certificat de civisme ${ }^{73}$, ce qui accroît encore la confusion entre l'arbitrage et les autres fonctions publiques de justice, en même temps que cette obligation permet de contrôler l'accès à la fonction arbitrale, laquelle ne pourra désormais être exercée que par des individus aux idées conformes à l'orthodoxie révolutionnaire ${ }^{74}$. Les arbitres montpellićrains prennent généralement soin de constater dans leur sentence que cette condition est remplie ${ }^{75}$.

(63) Décret du 12 brumaire an II, art. 18, DUVERGIER, op. cit., t. VI, p. 271.

(64) lbid., art. 3 à 7.

(65) Ihid., art. 18 al. 2.

(66) Décret des 17-21 nivôse an Il, art. 54, Duvergil:R, op. cit., t. VI. p. 380.

(67) Ibid., art. 55 al. 1 . Le décret du 23 ventôse an II vient ensuite limiter le nombre des arbitres à deux par intérêt en présence, DUvergiler, op. cit., t. VII, p. 105.

(68) Décret du 17 nivôse an II, art. 55 al. 2, Duvergilek, op. cit., t. VI, p. 381.

(69) $\mathrm{lbid}$., art. 55 al. 3.

(70) Loi du 9 fructidor an II, art. 8 et s., DuVErgIER, op. cit., t. VII, p. 255

(71) $\mathrm{lbid}$, art. $11 \mathrm{ct} 12$.

(72) Elles choisissent plutôt des notaires comme arbitres et en désignent deux par intérêt en présence : AD Hérault, L 6338, 8 germinal an II, Jugement arbitral entre les citoyens Barreau frère ct scurs.

(73) Décret du 6 germina! an II qui exige un certificat de civisme pour être arbitre, DUvERGIER, op. cit., t. VII, p. 123.

(74) Cf. Jean-Louis HaL.PÉRIN, L'impossible Code civil..., op. cit., p. 183. La formalité du certificat de civisme est abolie par le décret du 18 thermidor an III, Duvfrgifr, op. cit., t. VIII, p. 210.

(75) Cf. AD Hérault, L 6338, 11 germinal an II, Jugement arbitral entre François Ginesty, Joseph Ginesti et Marguerite Michel. 
Une fois ces arbitres désignés, la loi indique qu'ils doivent procéder à l'instruction "sommaire " du litige, ce qui laisse place, à Montpellier, à des pratiques variées témoignant de ce que l'arbitrage est de plus en plus souvent conçu comme une véritable procédure judiciaire. Ainsi les parties se soumettent parfois à un préliminaire de conciliation devant le juge de paix, avant d'aller, munies de leur extrait du procès-verbal de non conciliation, devant le tribunal arbitral, comme elles le feraient avant de se présenter devant toute autre juridiction étatique ${ }^{7}$. Il arrive également que les arbitres n'entendent plus les parties lors de leurs sessions, et se contentent de l'examen des pièces qui leur ont été remises pour se déterminer ${ }^{7}$. Quand les parties sont encore entendues, ce n'est généralement plus que pour éclaircir le sens de pièces contraires, et non comme précédemment dans le but de parvenir à une solution négociée entre les différents acteurs de l'arbitrage ${ }^{7 /}$. La pratique prend ainsi acte de l'évolution judiciaire de l'arbitrage, et l'instance arbitrale se trouve en conséquence à la fois figée et standardisée.

Lorsque les arbitres délibèrent, ils ont donc désormais l'obligation de suivre la loi, sans pouvoir juger en équité. Les sentences arbitrales se résument alors à des liquidations de successions, comme dans le partage entre les héritiers Branjon. Les arbitres reconstituent le patrimoine du défunt et calculent le montant de la reprise à laquelle la veuve peut prétendre. Elle demandait 3000 livres pour la dot reçue de ses parents, ainsi que 800 livres pour remboursement de ses habits de deuil et de son année de viduité. Les arbitres réduisent cette dernière prétention à 550 livres, " eu égard aux facultés [du défunt] et aux jouissances par lui léguées à sadite épouse ". Les arbitres ordonnent aussi que la somme de 3550 livres soit prélevée sur l'hérédité avant tout partage. La veuve réclame ensuite l'exécution de plusieurs legs contenus dans le testament de son défunt mari, et appliquant la loi, les arbitres déclarent le testament du défunt nul en vertu de l'article $1^{\text {er }}$ de la loi du 17 nivôse, sauf pour les legs qui parce qu'ils «n'excèdent point la moitié des fruits de l'entière hérédité ", peuvent être maintenus. Ils procèdent de même pour partager les biens entre les héritiers.

Sur un point, les arbitres prennent tout de même la liberté de reconnaître que la lettre de la loi se heurte à son esprit et choisissent de rendre une décision conforme à ce dernier, se fondant sans le dire sur l'équité. Le problème vient ici de ce que le défunt a cédé à son fils aîné qui est aussi son héritier institué, peu de temps avant sa mort, une certaine quantité de

(76) Cf. AD Hérault, L 6335, 5 avril 1793. Sentence arbitrale pour Causse Marie Sellier.

(77) Cf AD Hérault, L 6338, 11 germinal an II, Jugement arbitral François Ginesty, Joseph Ginesti et la citoyenne Marguerite Michel.

(78) Cf. AD Hérault, L 6335, 5 avril 1793, Sentence arbitrale pour Causse contre Marie Sellier. 
marchandises. Le fils lui-même reconnaît que cette cession doit être annulée et les arbitres conviennent qu'elle « présente une suspection d'autant plus grande que ledit Branjon père était pour lors, et depuis longtemps dans sa maladie grave dont il est décédé un mois après, qu'il paraît ainsi que c'est un avantage qu'il a voulu faire à son dit fils au préjudice de ses autres enfants, qu'un pareil acte doit par conséquent tomber dans le cas de la nullité prononcé par le susdit article $1^{\text {er }}$ de la loi du 17 nivôse an II [...]». Mais si la cession doit être annulée, tout ce que l'héritier a reçu de son père doit alors être rendu à la succession, ce qui pose des problèmes d'estimation, car entre la cession et l'instance arbitrale, le décret du maximum est venu rabaisser les marchandises "à une valeur infiniment moindre que celle qu'elles pouvaient avoir à l'époque de la succession ", et " si cette perte devait retomber en entier " sur le fils aîné, "son lot dans ledit partage serait plus qu'entièrement absorbé ; qu'aussi rien ne blesserait davantage l'égalité que la susdite loy a voulu introduire dans les partages entre enfants ». Les arbitres préfèrent donc estimer eux-mêmes la valeur des marchandises, en choisissant celle qu'elles avaient au moment de la cession.

Une fois réglée cette difficulté, les arbitres reconstituent l'état du patrimoine et les sommes que se doivent les héritiers entre eux, sur le fondement de la loi du 17 nivôse an II (6 janvier 1794), avant de préciser que le partage n'est pas tout à fait définitif car « les biens immeubles et meubles doivent encore être estimés par des experts et répartis en cinq lots de valeur égale ${ }^{79}$.

Il arrive en effet, et c'est un autre signe de l'assimilation croissante de l'arbitrage à une procédure judiciaire, que les arbitres aient de plus en plus souvent recours à des experts afin d'estimer le patrimoine à partager entre les successibles. Il s'agit là d'une pratique tout à fait nouvelle car traditionnellement, les parties qui s'adressent à des arbitres font le choix d'individus particulièrement compétents dans la matière du litige, ce qui évite en principe d'avoir en plus recours à des experts, et permet de simplifier la procédure, réduire les frais et les délais par rapport à un procès devant la justice étatique. Or en application des lois de l'an II, le carcan législatif est tel que les arbitres se contentent de mener la procédure, et renvoient à des experts chaque fois qu'il est nécessaire ${ }^{k 0}$. Cette pratique a l'inconvénient de provoquer souvent l'ajournement de la procédure arbitrale, le temps que les experts soient choisis et qu'ils remplissent leur mission $^{81}$.

(79) AD Hérault, L 6338, 23 ventôse an II, Premic̀re sentence arbiltrale qui ordonne l'estimation entre les héritiers Branjon.

(80) AD Hérault, L 6337, 24 ventôse an II, Jugement arbitral entre les citoyennes Bourreli sœeurs et la citoyenne Fournier.

(81) AD Hérault, L 6338, 20 germinal an II, Jugement de famille entre Beloury frères et sceurs contre Beloury fils. 
Les suites de l'arbitrage ne font encore que confirmer l'emprise grandissante du législateur sur l'institution. Ainsi et pour la première fois, les arbitres sont autorisés à statuer sur les frais des procédures de partage ${ }^{82}$. Également, les sentences ne sont pas soumises à appel ${ }^{\times 3}$, mais le recours en cassation est possible, car pour la Convention, il est " bon sans doute de laisser aux citoyens cette ressource contre les infractions formelles de la loi ${ }^{84}$, preuve supplémentaire de la soumission toujours plus étroite de l'arbitrage à la loi.

Lon se demande ainsi si les litiges réglés en grand nombre par les arbitres à la suite des lois de l'an II le sont véritablement par la voie de l'arbitrage. L'esprit d'abord est désormais très différent car il ne s'agit plus de permettre par la conciliation le rétablissement de la concorde au sein d'une famille en conflit, ce que pratique et volonté politique avaient pourtant souhaité jusque là ${ }^{\mathrm{ks}}$. Il est surtout difficile de reconnaître dans les arbitrages rendus depuis 1793 l'arbitrage tel que pratiqué jusque-là. Le fait notamment que les parties ne puissent plus directement ni indirectement choisir le tiers-arbitre, celui pourtant dont l'avis s'imposera face à des arbitres divisés ; le fait également que les arbitres n'aient plus la possibilité de se déterminer suivant l'équité, tendent à montrer que « l'arbitrage » n'est plus qu'un mot qui recouvre une réalité non plus extrajudiciaire mais « intrajudiciaire ", à l'intérieur de la justice d'État, dont il occupe désormais une partie de l'espace.

Certes, malgré de telles contraintes, il arrive encore parfois que parties et arbitres parviennent à mettre en place des stratégies de contournement de la loi, qui leur permettent dans une certaine mesure de reprendre en main l'instance arbitrale. Ainsi pour échapper aux règles de récusation et au contrôle du juge de paix, les parties précisent par exemple que les arbitres choisis ont été agréés par l'ensemble des parties ${ }^{\mathrm{\gamma}}$. Il arrive aussi parfois que les arbitres demandent encore aux parties de les éclairer sur le sens d'une preuve, cherchant par là à obtenir leur accord sur ce point $^{87}$. Les arbitres veulent aussi quand ils le peuvent discuter avec les litigants des modalités du partage ${ }^{88}$, et souhaitent toujours prendre en compte

(82) Loi du 9 fructidor an II, art. 7 al. 2, Duvergier, op. cit., t. VII, p. 255. Cf. par exemple AD Hérault, L 6338, 1 germinal an II, Jugement arbitral pour Françoise Plantade contre les héritiers naturels de Couderc.

(83) Décret des 17-21 nivôse an II, art. 56, DUVERGiler, op. cit., t. VI, p. 381.

(84) Décret du 22 ventôse an II, 60\% question, Duvergiter, op. cit., t. VII, p. 104-105.

(85) Voir pour une autre vision de cette question Jean-Jacques ClerE, "Larbitrage révolutionnaire... ", op. cit., p. 12.

(86) Cf. par exemple AD Hérault, L 6338, Première sentence arbitrale qui ordonne l'estimation entre les héritiers Branjon ; AD Hérault, $L$ 6338, $1^{\text {" }}$ germinal an II, Jugement arbitral pour François Plantade contre les héritiers naturels de Couderc.

(87) AD Hérault, L 6338, 20 germinal an II, Jugement de famille entre Beloury frères et sceurs contre Beloury fils.

(88) AD Hérault, L 6337, 24 ventôse an Il, Jugement arbitral entre les citoyennes Bourreli sceurs et la citoyenne Fournier. 
leurs propositions afin de parvenir à un meilleur règlement du litige ${ }^{k 9}$. Ainsi, par touches légères, parties et arbitres parviennent encore à insuffler un peu de cet esprit de conciliation qui semble tout de même globalement appartenir au passé.

L'arbitrage rendu en application des lois de l'an II a été souvent critiqué, y compris à son époque ${ }^{i / 1}$. Pourtant, au vu des archives montpelliéraines, il apparaît que les arbitres ont fait une application plutôt mesurée des lois nouvelles ${ }^{91}$. L'éloge du fond n'en emporte pas moins une critique de la forme, car il demeure que l'arbitrage peine à retrouver sa véritable nature ainsi qu'un mode de fonctionnement véritablement efficace. Une telle situation n'allait toutefois pas durer, et la Convention thermidorienne allait atteindre entre autres cibles celle de l'arbitrage forcé. Dès la Constitution du 5 fructidor an III (22 août 1795), il n'est plus question que d'arbitrage volontaire, auquel le pouvoir politique conserve toute sa faveur ${ }^{92}$. Logiquement, deux lois du 9 ventôse an IV (27 février 1796) viennent supprimer l'arbitrage forcé comme " contraire à la constitution et implicitement supprimé par elle $»^{4,3}$, ajoutons contraire même à sa véritable nature.

\author{
Carine JALLAMION \\ Université de Franche-Comté \\ UFR des sciences juridiques, \\ économiques, politiques et de gestion \\ $45 \mathrm{~d}$ avenue de l'Observatoire \\ 25030 Besançon cedex \\ Carinejallamion@hotmail.com
}

(89) AD Hérault, L 6338, $8^{\mathrm{c}}$ germinal an 11, Jugement arbitral entre les citoyens Barreau frère et sceurs.

(90) Voir Claudine Bloch et Jean Hilaire, op. cit., p. 477

(91) Voir en ce sens pour la Bourgogne les conclusions de Françoise ForTuNET, « Connaissance et conscience juridique à l'époque révolutionnaire en pays de droit coutumier : la législation successorale *, La Révolution et l'ordre juridique privé..., op. cit., t. I, p. 366.

(92) Constitution du 5 fructidor an III, art. $210 \mathrm{et} 211$, DUVERGIER, VIII, 223.

(93) Lois du 9 ventôse an IV, DUVERGILR, IX, 52. 